\title{
Caracterización de la respuesta en fase y compensación de fase en sistemas multiportadora para canales con respuesta en frecuencia no uniforme*
}

Claudia Lucía Cortés Cortés*

Neil Guerrero González ${ }^{* * *}$

Recibido: 11/01/2018 - Aceptado: 23/07/2019

https://doi.org/10.22395/rium.v19n36a8

\begin{abstract}
Resumen
En este trabajo se propone una metodología de estimación de fase por subbandas de un canal de respuesta no uniforme. La novedad de la propuesta radica en la utilización de un formato de modulación en fase en cuadratura QPSK, (Quadrature Phase Shift Keying) modificado como señal de prueba para la estimación de fase que elimina el problema de la ambigüedad. La metodología propuesta se verifica experimentalmente utilizando como canal de transmisión un cable de RF defectuoso, logrando la transmisión de datos a tasas de 20 Kbaud en una distancia de $120 \mathrm{~cm}$ en una banda de frecuencias comprendida entre los $150 \mathrm{kHz}$ y $276 \mathrm{kHz}$.
\end{abstract}

Palabras clave: modulación multiportadora; respuesta en frecuencia no plana-no uniforme; respuesta en fase;Nyquist FDM; QPSK no convencional.

* Artículo de investigación en curso. Proyecto de tesis doctoral titulado Metodología de diseño de formatos de modulación multiportadora adaptativos para canales variantes en el tiempo selectivos en frecuencia. Universidad Nacional de Colombia-Colciencias.

** Magíster en Ingeniería en Automatización Industrial, estudiante del Doctorado en Ingeniería Automática, Universidad Nacional de Colombia, Manizales, Colombia. Correo electrónico: clcortesc@unal.edu.co. Orcid: https://orcid.org/0000-0001-5760-9990

*** Doctor en Fotónica. Profesor asociado del Departamento de Ingeniería Eléctrica, Electrónica y Computación, Universidad Nacional de Colombia, Manizales, Colombia. Correo electrónico: nguerrerog@unal.edu.co. Orcid: https://orcid.org/0000-0002-7070-878X 


\title{
Characterization of the In-Phase Response and Phase Compensation in Multicarrier Systems for Channels with Non-Uniform Frequent
}

\begin{abstract}
This work proposes a methodology for the phase estimation in sub-bands of a non-uniform response channel. The novelty of this proposal lies in the use of a modulation format in QSPK (Quadrature Phase Shift Keying) quadrature-phase modified as a test signal for the phase estimation that eliminates the ambiguity issue. The methodology here proposed is verified experimentally using a defective RF cable as transmission channel and thus achieving data transmission rates of $20 \mathrm{Kbaud}$ in a $120 \mathrm{~cm}$ distance in a frequency band between the $50 \mathrm{kHz}$ y $276 \mathrm{kHz}$.
\end{abstract}

Keywords: multicarrier modulation; non-uniform frequency response; phase response; Nyquist - FDM; unconventional QPSK.

\section{Caracterização da resposta em fase e compensação de fase em sistemas multiportadoras para canais com resposta em frequência não uniforme}

\begin{abstract}
Resumo
Neste trabalho, propõe-se uma metodologia de estimativa de fase por subfaixas de um canal de resposta não uniforme. A novidade da proposta está na utilização de um modelo de modulação em fase de quadratura QPSK (Quadrature Phase Shift Keying) modificado como sinal de teste para estimar a fase que elimina o problema da ambiguidade. A metodologia proposta é verificada de forma experimental com a utilização de um cabo de RF defeituoso como canal de transmissão, o que faz com que haja a transmissão de dados a taxas de $20 \mathrm{Kbaud} \mathrm{em} \mathrm{uma} \mathrm{distância} \mathrm{de} 120 \mathrm{~cm}$ em uma faixa de frequências compreendida entre $150 \mathrm{kHz}$ e $276 \mathrm{kHz}$.

Palavras-chave: modulação multiportadora; resposta em frequência não plana-não uniforme; resposta em fase; Nyquist FDM; QPSK não convencional.
\end{abstract}




\section{INTRODUCCIÓN}

La demanda creciente por velocidades mayores de envío y descarga de archivos en conexiones estables por parte de los usuarios de servicios de telecomunicaciones inalámbricas, han incrementado los requerimientos tanto en el diseño como en la implementación de nueva infraestructura de telecomunicaciones [1].

En efecto, con el fin de responder a las demandas de ancho de banda, se ha propuesto desde la literatura científica la creación de infraestructuras híbridas donde convergen sistemas de telecomunicaciones históricamente independientes, como los sistemas de transmisión por fibra óptica, por medio inalámbrico o cables coaxiales [2]. En este sentido, durante la primera década del siglo XXI los sistemas de radio sobre fibra (RoF, Radio overFiber) fueron ampliamente estudiados precisamente por el aumento significativo del ancho de banda que podía ser utilizado. Adicionalmente, la inclusión de fibra óptica como medio de transporte de datos entre las antenas emisorasreceptoras (estaciones base), extendió considerablemente la cobertura del servicio de telecomunicaciones [3]. Uno de los problemas de investigación más significativos durante el diseño de los primeros sistemas RoF, radicó en la compensación de los efectos de canal que sufría la señal de información durante las etapas de conversión eléctrico-óptico-eléctrico inherentes a la transmisión por RoF [4]. Como soluciones propuestas, se desarrollaron sistemas de estimación y compensación del canal híbrido óptico-eléctrico implementados como funciones de los receptores, como lo fueron tanto los ecualizadores asistidos por datos o tramas de entrenamiento como los ecualizadores ciegos [5], [6].

Con la llegada de los sistemas de RoF, la comunidad científica se enfrentó a un nuevo paradigma en cuanto a la concepción de modelo de canal de transmisión enseñado hasta hoy en las facultades de ingeniería. La convergencia de redes de telecomunicaciones en una plataforma única como la que soportará los nuevos servicios enmarcados en el 5G, exige la investigación de nuevos esquemas de estimación y compensación de efectos de canales generalizados durante la transmisión de datos, incluyendo canales de respuesta en frecuencia no uniforme, selectivos en banda y variantes en el tiempo [7].

Esta generalización del modelo de canal de transmisión incluyendo respuestas en frecuencia no uniforme, introduce nuevos desafíos en el diseño de sistemas de ecualización, como la necesidad de estimaciones de canal a tramos o por subbandas con el fin de maximizar la eficiencia espectral en el canal. Entre los diferentes tipos de estimadores, se destacan el estimador de máxima verosimilitud (MaximumLikelihood, ML), estimador de error cuadrático medio mínimo (Minimum Mean Squared Error, MMSE), estimador de Mínimos Cuadrados (LeastSquares, LS), y el estimador EM (ExpectationMaximization) [8], [6]. También se pueden usar algunos esquemas de 
interpolación en una o dos dimensiones, y en el dominio del tiempo o de la frecuencia para conocer la respuesta del canal [9].

Por un lado, la aproximación de estimación por subbandas permite el diseño de formatos de modulación multiportadora, donde las portadoras se adaptan tanto a la relación señal a ruido (SNR, signaltonoise ratio) presente en cada subbanda, como al desfase asociado a la misma. Esta necesidad de adaptación de cada portadora componiendo el formato multiportadora, dificulta la implementación de otros tipos de formatos como el OFDM (Orthogonal Frequency Division Multiplexing), debido a que la generación simultánea de todas las portadoras ortogonales en el dominio de la frecuencia a partir de la operación de la IFFT (transformada inversa de Fourier), imposibilita la modificación de los parámetros de cada portadora (formato de modulación o desfase) de forma independiente [10], [11]. Recientemente, se han presentado en la literatura esquemas de multiplexación basados en formas de pulso SINC que generan espectros de potencia cuasi rectangulares. Estos esquemas de eficiencias espectrales mayores son conocidos como multiplexación en el dominio de la frecuencia Nyquist [12].

Por otro lado, la estimación de fase con propósitos de precompensación ha sido ampliamente reportada en la literatura, siendo el algoritmo de Viterbi-Viterbi el más popular hasta nuestros días [13]. Sin embargo, la remoción de la información de fase de la señal de información a partir de la aplicación de operaciones de potencia (elevación al cuadrado, o a la cuarta potencia) asociada a los algoritmos de estimación de fase, genera errores de estimación debido a laimposibilidad dedetectar desfases en el rango completo comprendido entre 0 y 360 grados. Este problema es también conocido como fase ambigua [14], [15].

En este trabajo proponemos una metodología de estimación de fasepor subbandas de un canal de respuesta no uniforme como se ilustra en la figura 1. La novedad de la propuesta radica en la utilización de un formato modificado de modulación en fase en cuadratura QPSK(Quadrature Phase Shift Keying) aplicado a la trama de entrenamiento (o tono piloto), que elimina el problema de la fase ambigua y permite la precompensación de fase de las portadoras. Adicionalmente, el tono piloto presenta un espectro cuasi rectangular debido a la implementación de formas de pulso SINC. La modificación del formato QPSK consiste en la alteración del módulo constante de las modulaciones PSK, al disminuir de manera controlada la potencia de uno de los cuatro símbolos del formato, permitiendo la estimación de la fase en el rango completo comprendido entre los 0 y 360 grados. El proceso de estimación de fase se basa en la aplicación del algoritmo de los K-medios (K-means) sobre los símbolos ubicados en el plano IQ de la constelación, cuyo propósito es encontrar tanto el valor esperado de potencia de cada símbolo QPSK modificado como la fase asociada a cada símbolo. 


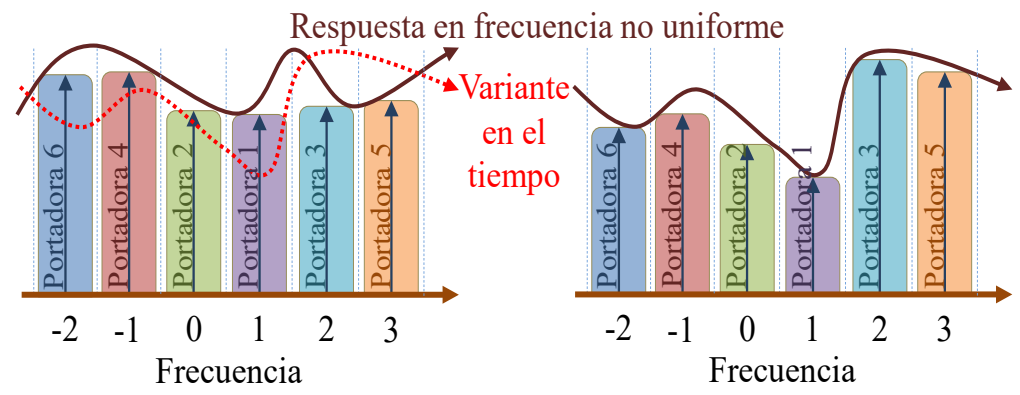

Figura 1. Espectro de frecuencia de una señal con múltiples portadoras adaptadas a la respuesta en frecuencia no uniforme del canal Fuente: elaboración propia

Con el fin de verificar el desempeño del método propuesto, se implementa en un primer momento una plataforma de simulación de respuesta no uniforme a partir de la respuesta en fase lineal de filtros FIR y la respuesta en fase no lineal de filtros IIR. En un segundo momento, se lleva a cabo una validación experimental utilizando como canal un cable de RF defectuoso, y se logra la transmisión de datos a tasas de 20 Kbaud por una distancia de $120 \mathrm{~cm}$ en una banda de frecuencias comprendida entre los $150 \mathrm{kHz}$ y $276 \mathrm{kHz}$.

El presente artículo se divide en cinco secciones. En la sección 1 se presenta el marco teórico que sustenta los elementos base de la propuesta metodológica. La descripción paso a paso de la propuesta metodológica se presenta en la sección 2, para luego mostrar en la sección 3 los resultados en simulación. Por último, en la sección 4 se encuentra la validación experimental de la propuesta metodológica, y las conclusiones se detallan en la sección 5 .

\section{PROPUESTA METODOLÓGICA: MARCO TEÓRICO}

En esta sección se presenta el marco teórico que sustenta la propuesta metodológica, compuesto por tres elementos base. Primero se deberá diseñar un esquema de modulación multiportadora Nyquist-FDM, donde cada portadora tendrá una forma cuasi rectangular en el dominio de la frecuencia. Para realizar la estimación del canal se diseñará un esquema de modulación QPSK no convencional por portadora, el cual permitirá identificar la rotación de los símbolos en el diagrama de constelación de la señal recibida. Finalmente, el uso del algoritmo de K-medias como herramienta de diagnóstico permitirá identificar, ubicar y calcular la variación en la fase de los símbolos en el diagrama de constelación. Esta información será de utilidad para precompensar el desfase asociado a cada subbanda evaluada en el modulador. 


\subsection{Modulación multiportadora Nyquist - FDM}

Esta modulación consiste en el uso de un filtro RRC (Root Raised Cosine) para llevar a cabo la operación de formación de pulsos (pulse shaping),generando formas de onda de tipo SINC sobre cada uno de los bits que serán transmitidos y la subsecuente generación de la señal moduladora [16]. La aplicación del filtro RRC permite la variación del parámetro conocido como factor de roll-off que define el ancho de banda que será ocupado por la señal moduladora. Así, un valor de roll-off de 0,1 creará una señal moduladora con un espectro cuasi-rectangular cuyo ancho de banda será 1,1 veces el valor de la tasa de bits [17]. Tanto un espectro cuasi rectangular, como la posibilidad de reducir a la mitad el ancho de banda de la señal moduladora,comparado con el uso de pulsos rectangulares en lugar de forma de pulsos SINC, permite doblar la eficiencia espectral al momento de generar un formato multiportadora (figura 2).
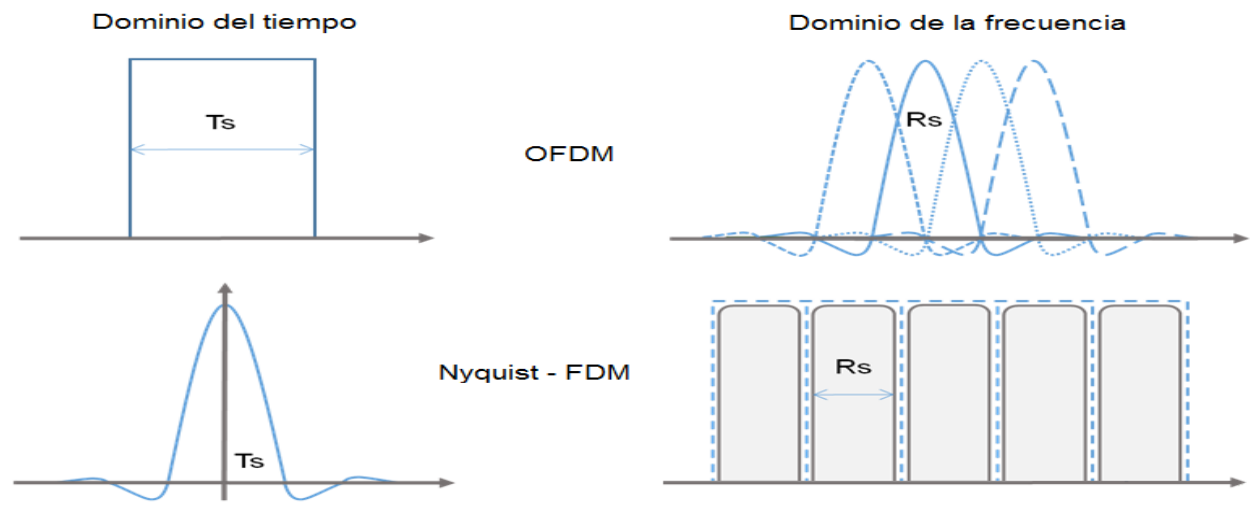

Figura 2. Comparación entre OFDM y Nyquist-FDM en el dominio del tiempo (izquierda) y en el dominio de la frecuencia (derecha)

Fuente: elaboración propia

Como consecuencia, el uso de este esquema de modulación multiportadora permitirá teóricamente duplicar el número de portadoras para transmitir la información al hacer un uso más eficiente del espectro, y discretizar el espectro permitiendo tener control sobre cada una de las portadoras. Asimismo, la implementación de la modulación multiportadora Nyquist-FDM, permitirá obtener mayor número de muestras del canal para caracterizar las variaciones de su respuesta en fase. 


\subsection{Modulación QPSK no convencional}

Esta es una modulación en fase cuya diferencia primordial respecto de una modulación QPSK convencional es la modificación del módulo constante, disminuyendo el módulo de un símbolo predeterminado (figura 3 izquierda). La modificación del módulo de un símbolo permite identificar la rotación de la constelación de la señal recibida (figura 3 centro y derecha), y compensar corrimientos en la fase de detección de los símbolos [1].
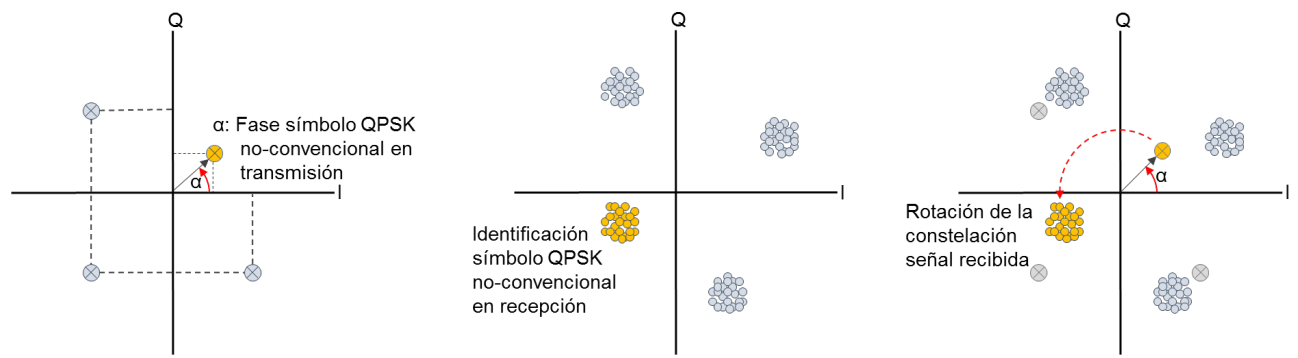

Figura 3. Diagrama de constelación modulador QPSK no convencional (izquierda), diagrama de constelación señal QPSK no convencional recibida (centro) y comparación del diagrama de constelación en transmisión y recepción (derecha) Fuente: elaboración propia

El desplazamiento de los símbolos a un cuadrante diferente al cuadrante original usado para generar el símbolo complejo en el diagrama de constelación se denomina Cycle Slip.Si la secuencia de errores es relativamente larga, estos pueden no ser corregidos usando corrección de errores hacia adelante - Forward-Error Correction-, el cual tiene usualmente una capacidad de corrección de errores limitada [18].

\subsection{Variación en fase: algoritmo K-medias}

El algoritmo de agrupamiento K-medias se implementó para calcular los corrimientos en fase en el diagrama de constelación de la señal recibida. K-medias es un algoritmo de agrupamiento que requiere un número inicial de grupos (Cluster) especificado por el programador, el cual para el caso de un modulador QPSK serán siempre cuatro. Cada uno de los puntos presentes en el diagrama de constelación es asignando al centro más cercano. Luego el valor de los centros se actualiza a medida que se van incorporando nuevos puntos al grupo. Como se presenta en la figura 4, una vez que todos los puntos presentes en el diagrama de constelación han sido asignados a uno de los cuatro K-grupos, se inicia un proceso iterativo para calcular los centroides finales de esos K-grupos [19], [20]. 
Posteriormente, mediante una comparación entre los valores de los centroides de los cuatro K-grupos, se selecciona el valor del centroide correspondiente al símbolo de menor módulo. El cálculo de la variación de fase es una operación aritmética entre el valor del centroide seleccionado y el valor del símbolo de módulo menor programado en el modulador QPSK no convencional. Este proceso se debe realizar con cada una de las portadoras para obtener la respuesta en fase del canal.

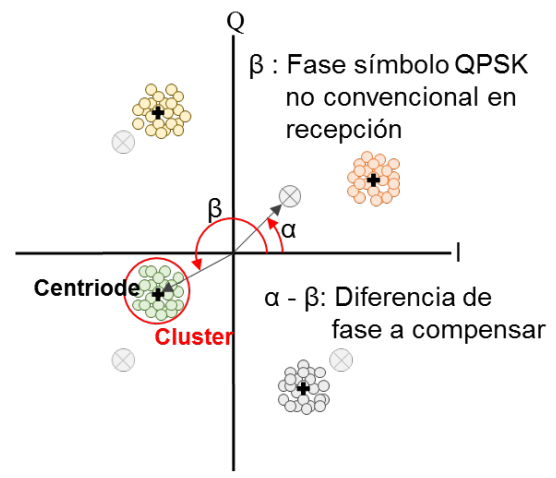

Figura 4. Aplicación algoritmo K-means para ubicación los símbolos en el diagrama de constelación de la señal QPSK no convencional recibida Fuente: elaboración propia

\section{DESCRIPCIÓN DE LA PROPUESTA METODOLÓGICA}

En esta sección se presentan los pasos para estimar las variaciones en fase por subbanda de un canal con respuesta en frecuencia no uniforme según la propuesta metodológica planteada. La metodología propuesta se describe paso a paso y se presenta su respectivo diagrama de flujo en la figura 5.

- Paso 1: dividir el ancho de banda del canal disponible para transmisión en subbandas equiespaciadas de $21 \mathrm{kHz}$.

- Paso 2: diseñar un modulador QPSK no convencional como se describe en el apartado 1.2, cuyas portadoras tendrán una frecuencia central como se definió en el paso 1. Luego, transmitir la señal modulada usando un modulador QPSK no convencional a través del canal de transmisión.

- Paso 3: estimarla diferencia de fase entre la señal recibida modulada usando un modulador QPSK no convencional, usando el algoritmo de agrupamiento y almacenar dicho valor.

- Paso 4: repetir el paso 2 y el paso 3 hasta realizar la estimación de fase para cada portadora cuya frecuencia central fue definida en el paso 1. 


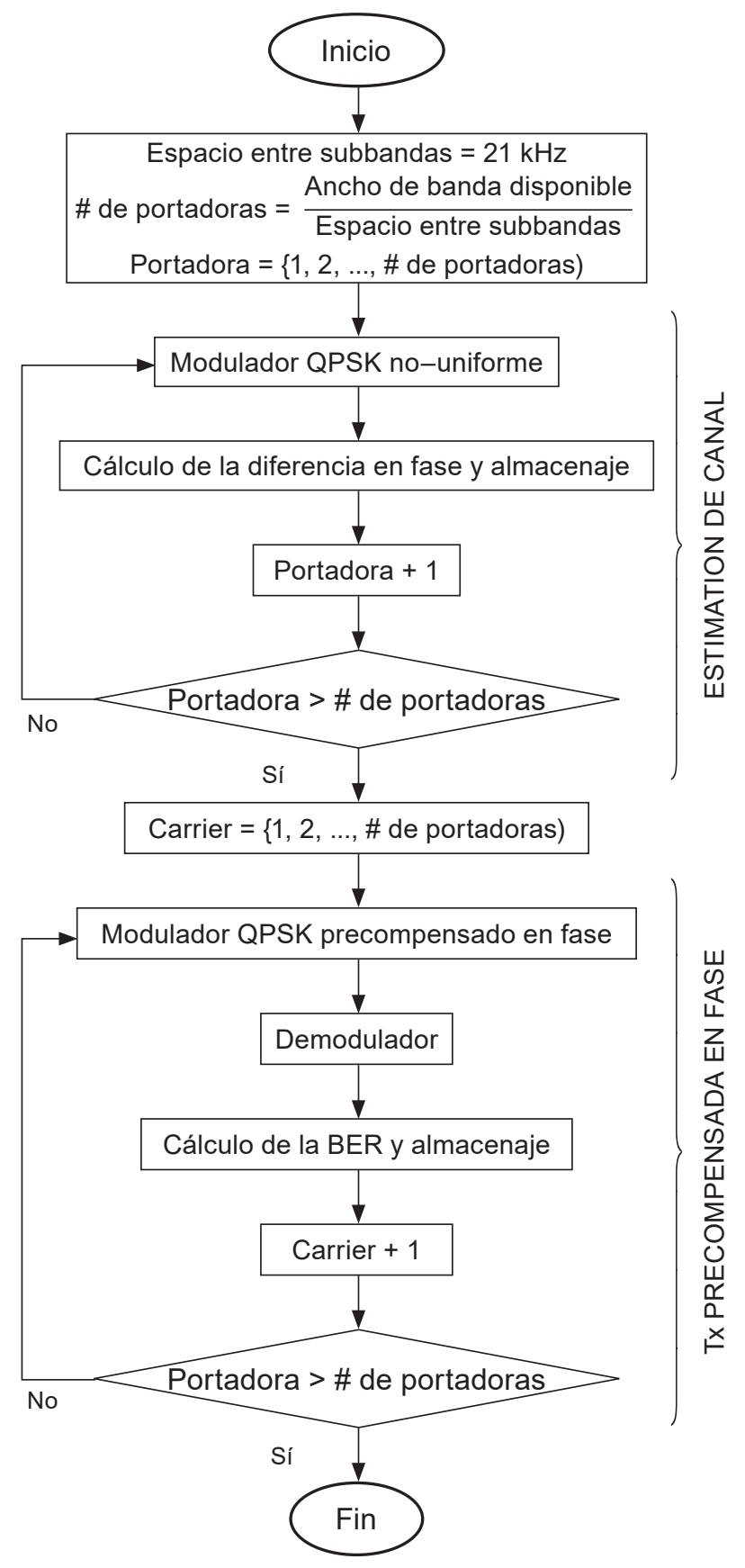

Figura 5. Diagrama de flujo propuesta metodológica Fuente: elaboración propia 
- Paso 5: configurar un modulador QPSK y precompensarla fase según se calculó en el paso 3 para las portadoras definidas en el paso 1. Luego se transmite la señal modulada usando un modulador QPSK precompensado en fase a través del canal de transmisión.

- Paso 6: usar un demodulador QPSK convencional, calcular la tasa de error de bit $\mathrm{y}$ almacenar dicho valor.

- En el diagrama de constelación de la señal recibida se podrá observar que la rotación en fase de los símbolos se corrigió, lo que permitirá usar un demodulador QPSK y recuperar los datos sin errores.

- Paso 7: repetir el paso 4 hasta el paso 6 y realizar el cálculo de la tasa de error de bit para cada portadora cuya frecuencia central fue definida en el paso 1 .

\section{RESULTADOS EN SIMULACIÓN}

La caracterización de la respuesta en fase de un canal con respuesta en frecuencia no uniforme, fue evaluada mediante simulación usando datos generados de forma aleatoria. Para emular el canal se usó un filtro pasabanda entre el transmisor y el receptor, se usaron filtros pasabanda IIR (figura 6) de orden 3 diseñados a partir de prototipos analógicos (Butterworth y Elíptico), los cuales tienen una respuesta en fase no lineal y FIR (figura 7) de orden 200 basados en el método de ventanas (Bartlett y Han), cuya respuesta en fase es lineal.

Las características propias de cada uno de los filtros permitieron atenuar y desfasar la señal transmitida, lo cual se evidencia usando seis portadoras de características fijas, equiespaciadas, ubicadas ocupando la banda de paso de cada uno de los canales (filtros) entre $150 \mathrm{kHz}$ y $276 \mathrm{kHz}$.Cada una de las portadoras posee las mismas características, estas son:

- Ancho de banda $20 \mathrm{kHz}$

- Forma de pulso Root Raised Cosine

- Modulación QPSK (no convencional para entrenamiento, y compensada en fase para verificación de la corrección en fase y cálculo de la BER)

- Frecuencia central por portadora programada en: $161 \mathrm{kHz}, 182 \mathrm{kHz}, 203 \mathrm{kHz}, 224$ $\mathrm{kHz}, 245 \mathrm{kHz}$ y $266 \mathrm{kHz}$ 


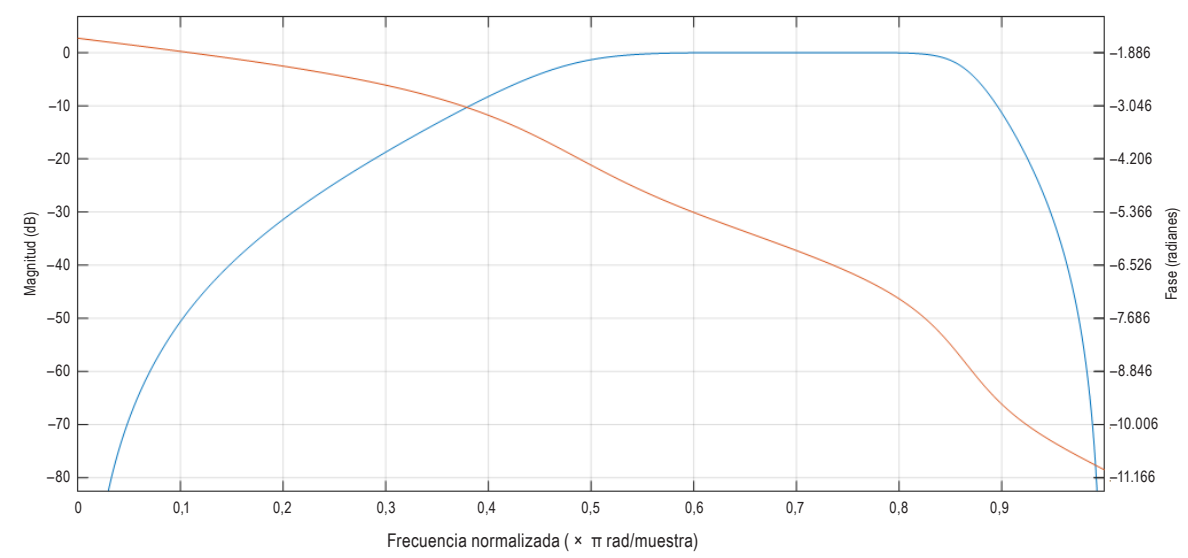

Figura 6. Respuesta en magnitud y fase de un filtro pasa banda IIR Butterworth Fuente: elaboración propia

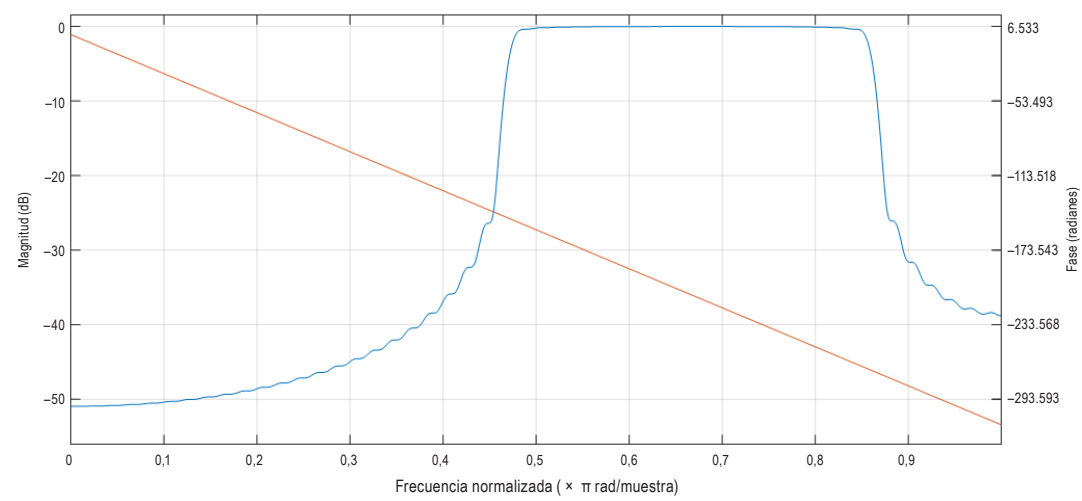

Figura 7. Respuesta en magnitud y fase de un filtro pasa banda FIR Bartlett Fuente: elaboración propia

Con los parámetros anteriores y siguiendo la metodología propuesta en la sección 2 se simularon dos transmisiones. En la primera se generaron 8.152 datos aleatorios que luego fueron modulados usando QPSK no convencional descrito en la sección 1.2. La señal modulada se transmitió a través del canal con respuesta en frecuencia no uniforme propuesto. Los diagramas de constelación obtenidos para cada portadora transmitida a través de un canal (filtro Butterworth) se presentan en la figura 8.

Como se observa en la figura 8 , el diagrama de constelación de cada portadora presenta una rotación diferente. En seguida, aplicando el algoritmo de K-medias sobre la señal recibida, se selecciona el centroide del símbolo de módulo de menorpotencia en cada portadora (figura 9) y se realiza el cálculo de la variación de fase. 


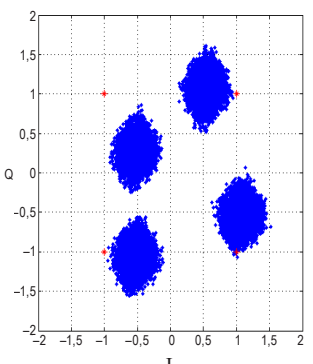

$161 \mathrm{kHz}$

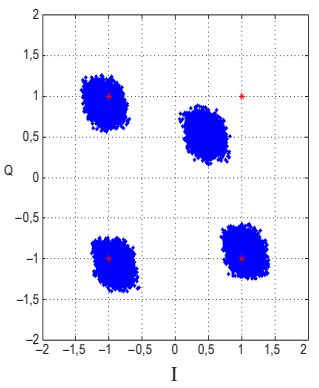

$224 \mathrm{kHz}$

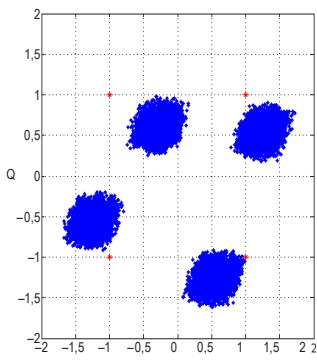

$182 \mathrm{kHz}$

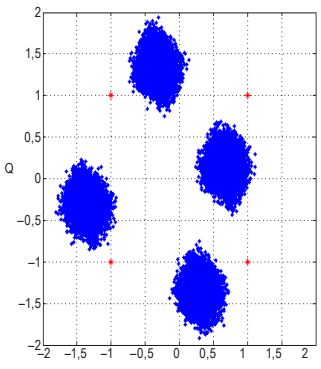

$245 \mathrm{kHz}$

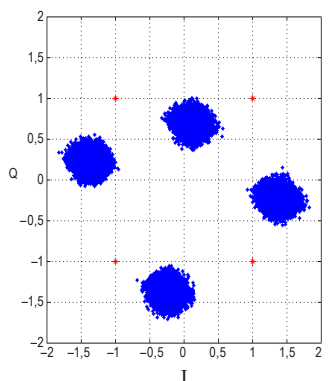

$203 \mathrm{kHz}$

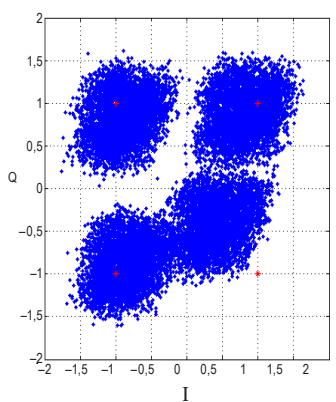

$266 \mathrm{kHz}$

Figura 8. Diagrama de constelación recibido para las seis portadoras equiespaciadas Fuente: elaboración propia

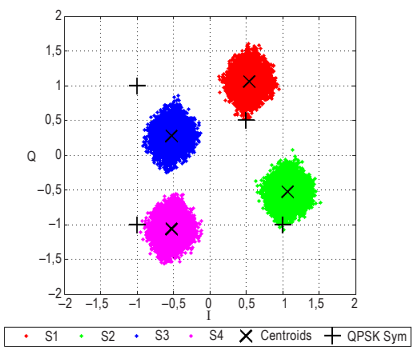

$161 \mathrm{kHz}$

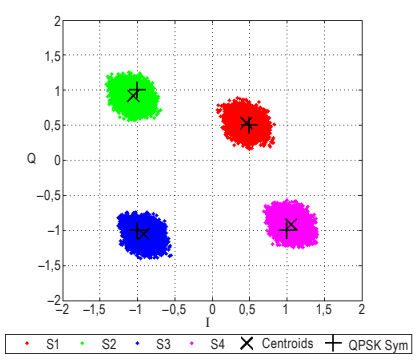

$224 \mathrm{kHz}$

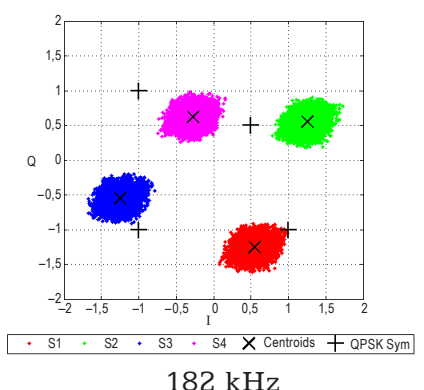

$182 \mathrm{kHz}$

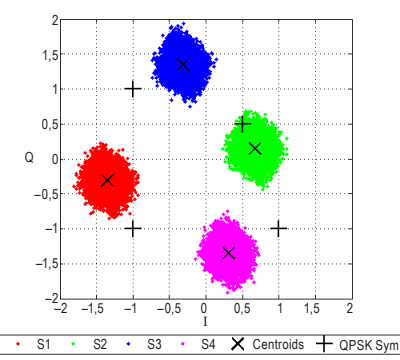

$245 \mathrm{kHz}$
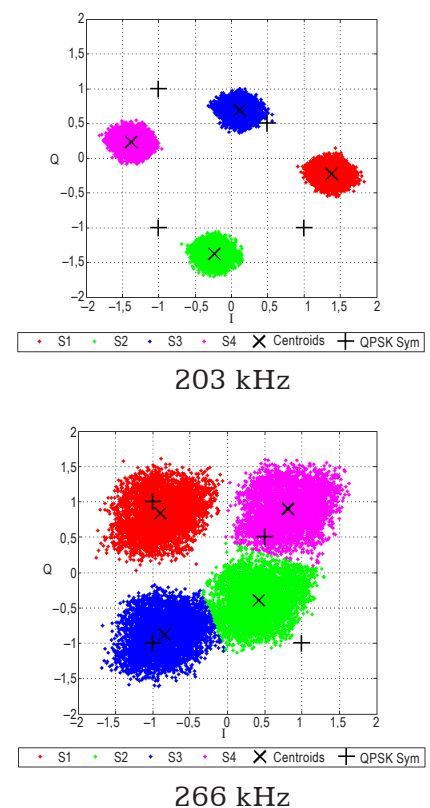

$266 \mathrm{kHz}$

Figura 9. Ubicación de los centroides usando el algoritmo K-medias

Fuente: elaboración propia 
Finalmente, usando como insumo la diferencia de fase calculada entre el valor del centroide seleccionado y el valor del símbolo de módulo de menor potencia en el modulador QPSK no convencional, se realizó una segunda transmisión. En esta se configuró un modulador QPSK precompensado en fase. En la figura 10 se presentan los diagramas de constelación de las señales QPSK precompensadas en el lado del receptor.

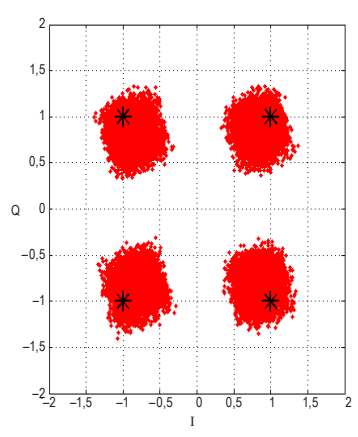

$161 \mathrm{kHz}$

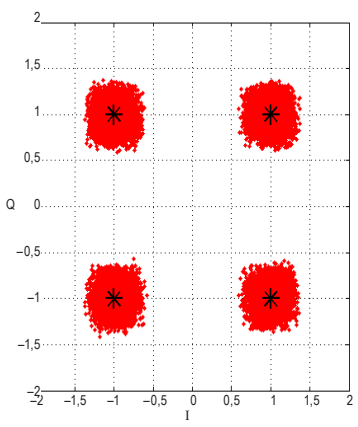

$224 \mathrm{kHz}$

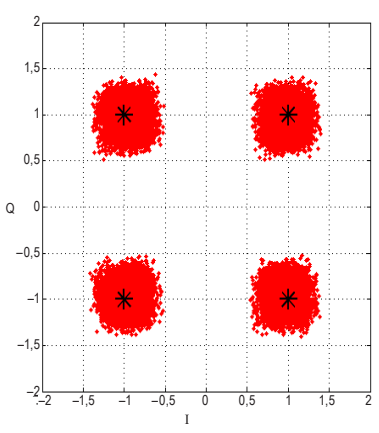

$182 \mathrm{kHz}$

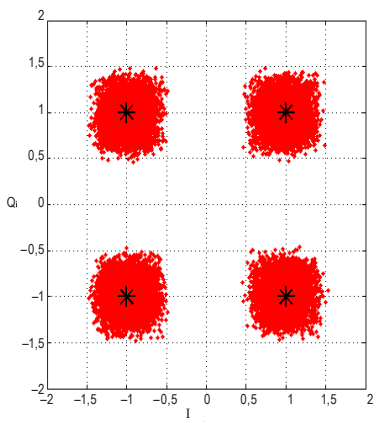

$245 \mathrm{kHz}$

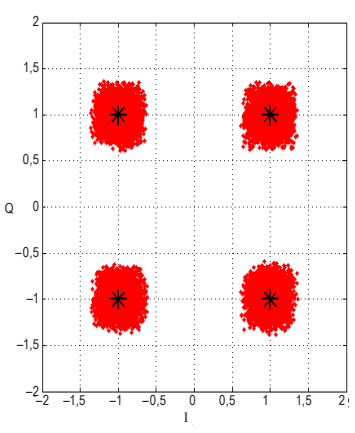

$203 \mathrm{kHz}$

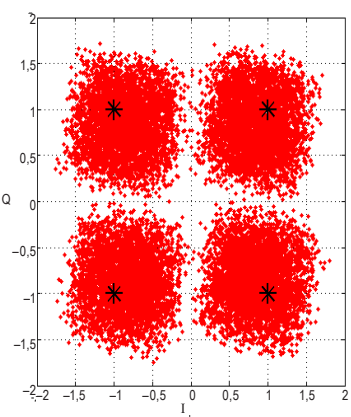

$266 \mathrm{kHz}$

Figura 10. Diagrama de constelación paso 6 de la metodología. Señal recibida luego de usar un modulador QPSK convencional compensado en fase Fuente: elaboración propia

Consecutivamente se realizó la demodulación de la señal, la cual se pudo realizar mediante un demodulador QPSK sin modificaciones. Tanto los diagramas de constelación presentados en la figura 10 como el cálculo de la BER indican la exitosa reubicación de cada uno de los símbolos en sus respectivos cuadrantes originales. Los valores dela BER obtenidos se presentan en la tabla 1. 
Tabla 1. BER calculado por portadora

\begin{tabular}{|l|c|c|c|c|c|c|}
\hline Frecuencia Central & $161 \mathrm{kHz}$ & $182 \mathrm{kHz}$ & $203 \mathrm{kHz}$ & $224 \mathrm{kHz}$ & $245 \mathrm{kHz}$ & $266 \mathrm{kHz}$ \\
\hline BER & 0 & 0 & 0 & 0 & 0 & $4,89 \times 10-4$ \\
\hline
\end{tabular}

Fuente: elaboración propia

Este proceso se repitió para cuatro filtros pasa banda cuya respuesta en fase estimada mediante modulación multiportadora Nyquist-FDM, varía de forma diferente respecto a la respuesta en fase estimada mediante la herramienta de diseño de filtros en el software MATLAB . En la figura 11 se observa claramente que la respuesta en fase caracterizada mediante modulación multiportadora Nyquist-FDM provee en detalle los cambios en fase en la banda de paso de cada uno de los filtros evaluados.

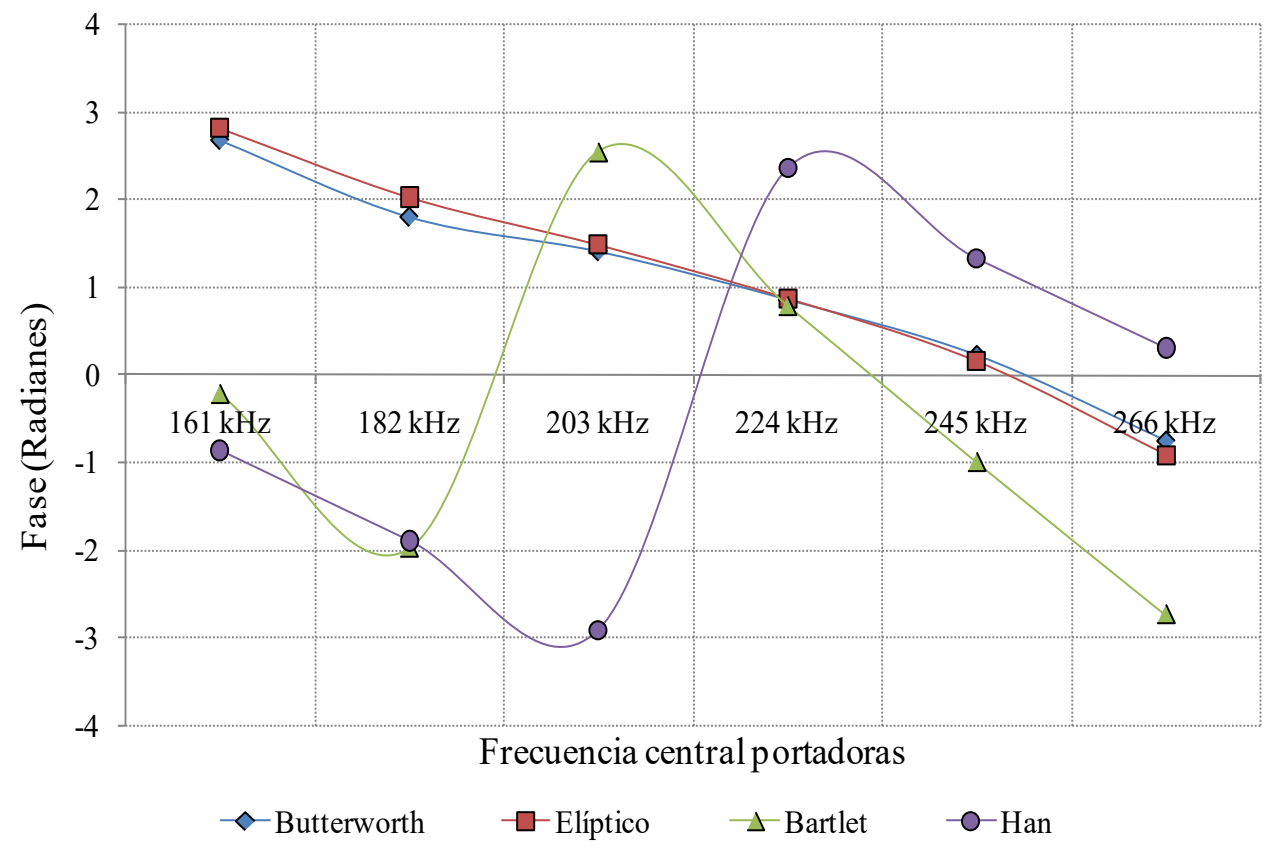

Figura 11. Respuesta en fase estimada mediante modulación multiportadora Nyquist-FDM Fuente: elaboración propia

En el caso de los filtros FIR evaluados - Bartlett y Han - cuya respuesta en fase debería ser lineal según la literatura, se observa un comportamiento no lineal, que para cada una de las pruebas realizadas el cálculo del BER dio como resultado cero en simulación.

El valor del BER obtenido para los filtros IIR evaluados - Butterworth y Elipticovarió entre 0 y $4,89 \times 10^{-4}$ en simulación, esto como se puede observar en el diagrama 
de constelación de la portadora cuya frecuencia central se ubica en $266 \mathrm{kHz}$ se debe a la interferencia entre los símbolos.

\section{VALIDACIÓN EXPERIMENTAL}

Con el propósito de validar la propuesta metodológica para estimación de la respuesta en fase por portadora de un canal, se realizaron tres pruebas experimentales. Los elementos usados para realizar el experimento fueron un osciloscopio de dominio mixto Tektronix MDO3012, un computador equipado con el software Matlab ${ }^{\circledR}$ que realiza las funciones de modulador y demodulador, y un cable BNC defectuoso. Integrado al Tektronix MDO3012 se encuentra un generador arbitrario de señales, el cual mediante Matlab ${ }^{\circledR}$ puede generar la señal modulada usando un modulador QPSK no convencional para realizar la estimación de la respuesta en fase por portadora. El cable BNC defectuoso, conectado entre la salida del generador arbitrario de onda y la entrada del osciloscopio, emula un canal variante en el tiempo como se presenta en la figura 12.

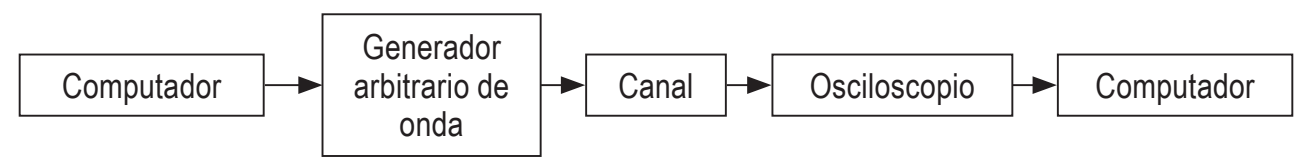

Figura 12. Diagrama de bloques del experimento

Fuente: elaboración propia

Se programaron mediante software seis portadoras, cada una con un ancho de banda de $20 \mathrm{kHz}$ y una frecuencia central ubicada en: $161 \mathrm{kHz}, 182 \mathrm{kHz}, 203 \mathrm{kHz}$, $224 \mathrm{kHz}, 245 \mathrm{kHz}$ y $266 \mathrm{kHz}$, para evaluar la propuesta metodológica de estimación de la respuesta en fase por portadora. Adicionalmente, por portadora se adaptaron algunas variables del código desarrollado en Matlab ${ }^{\circledR}$, realizando una exitosa transmisión usando el MDO3012: se transmitieron 1.024 símbolos, más 200 ceros para sincronización por portadora y se seleccionó una frecuencia de muestreo de $1 \mathrm{MHz}$.

Como se muestra en la metodología propuesta, se realizan dos transmisiones: la primera para estimación de la respuesta en fase por portadora usando un modulador QPSK no convencional, y la segunda para transmitir una señal precompensada en fase usando un modulador QPSK. En la figura 13 (izquierda) se presenta el diagrama de constelación de la señal recibida en la primera transmisión, la señal modulada usando un modulador QPSK no convencional y la ubicación de los centroides usando el algoritmo K-medias; en la figura 13 (derecha) se presenta el diagrama de constelación de la señal recibida usando un modulador QPSK precompensado en fase. 

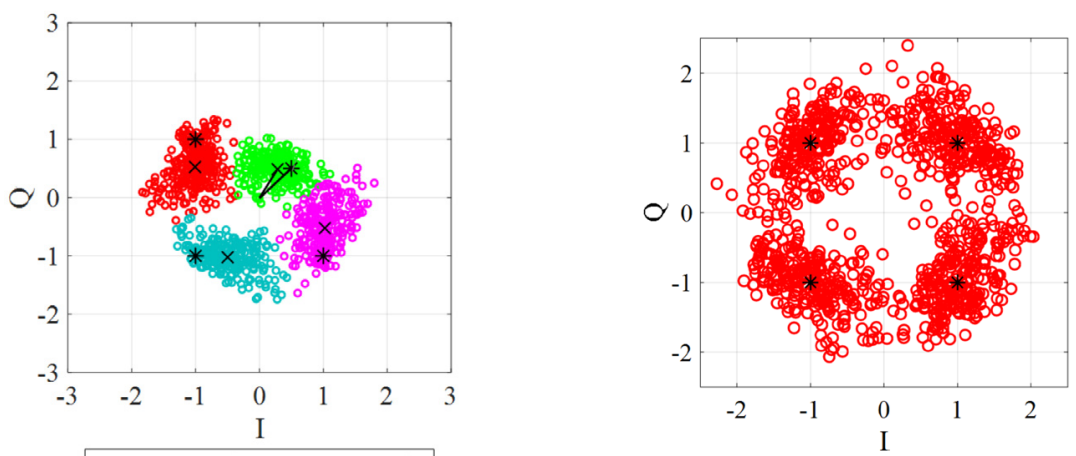

Figura 13. Diagrama de constelación de la señal recibida para la portadora ubicada en $161 \mathrm{kHz}$ : (izquierda) usando modulador QPSK no convencional y (derecha) usando modulador QPSK precompensado en fase

Fuente: elaboración propia

Los diagramas de constelación de la señal recibida por portadora y la tasa de error de bit calculada presentada en la tabla 2, indican la exitosa corrección de la fase de los símbolos dada la diferencia porcentual calculada por portadora.

Tabla 2: BER calculado por portadora señal sin compensar y señal precompensada

\begin{tabular}{lcccccc}
\hline Frecuencia central & $161 \mathrm{kHz}$ & $182 \mathrm{kHz}$ & $203 \mathrm{kHz}$ & $224 \mathrm{kHz}$ & $245 \mathrm{kHz}$ & $266 \mathrm{kHz}$ \\
\hline BER señal sin precompensar & 0,7588 & 0,7486 & 0,7497 & 0,7538 & 0,7637 & 0,7658 \\
\hline BER señal precompensada & 0,0420 & 0,0709 & 0,1196 & 0,1508 & 0,1932 & 0,2591 \\
\hline
\end{tabular}

Fuente: elaboración propia

Además la metodología propuesta también permite conocer la respuesta en fase del canal por portadora como se presenta en la figura 14. Aunque cabe resaltar que los resultados exactos no podrán ser reproducidos por causa de la falta de control sobre las variaciones del cable $\mathrm{BNC}$ defectuoso usado como canal, estas variaciones son para los autores el ambiente ideal para realizar la validación de la metodología propuesta. La estimación de la respuesta en fase por portadora para tres pruebas realizadas con un intervalo de treinta minutos entre pruebas es presentado.

Aunque se presentan errores por portadora, la propuesta metodológica permite recuperar entre $66 \%$ y $94 \%$ de los símbolos transmitidos por portadora usando un algoritmo de precomensación muy simple. 


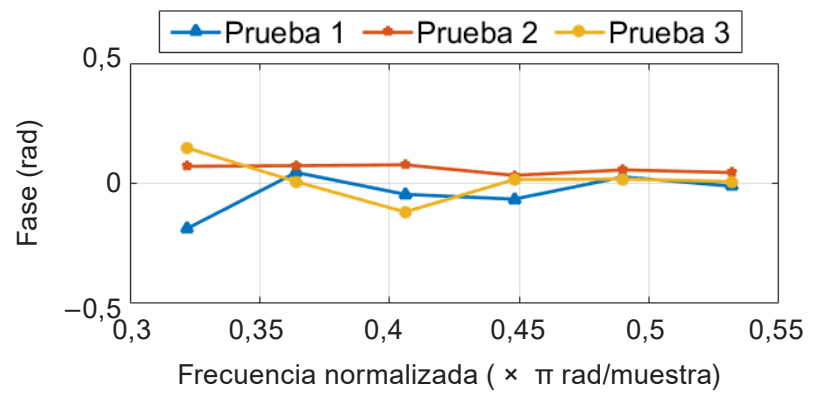

Figura 14. Respuesta en fase calculada para el canal emulado Fuente: elaboración propia

\section{CONCLUSIONES}

Se propuso una metodología para la caracterización de la respuesta en fase de un canal, mediante la implementación de un esquema de modulación multiportadora Nyquist-FDM siendo este uno de sus principales aportes.

La metodología propuesta posee varias ventajas respecto a las actuales técnicas para estimación de canal, entre ellas se encuentran: i) su fácil implementación en simulación gracias al uso de herramientas de cálculo y visualización intuitivas como lo son los diagramas de constelación y el algoritmo K-medias, ii) el incremento en el número de portadoras del esquema multiportadora, gracias a la forma de pulso Nyquist la cual puede llegar a duplicar la eficiencia espectral respecto de otros esquemas multiportadoracomo OFDM, a mayor número de portadoras mayor detalle en la caracterización, iii) la precisión en el cálculo de la fase al hacer uso del algoritmo K-medias y el ahorro en tiempo de cálculo respecto de las técnicas para estimación de canal clásicas, y iv) la facilidad para compensar la fase en el modulador y obtener un BER $=0$ en cada una de las portadoras, sin presencia de interferencia entre símbolos, lo que permitiría implementar el sistema de modulación adaptativo como trabajo futuro.

\section{AGRADECIMIENTOS}

Los autores agradecen el apoyo financiero a el proyecto DIMA 39053 Implementación y puesta en marcha de un Sistema de Generación Solar Fotovoltaica y Gestión Inteligente de Demanda, aprobado en la convocatoria nacional para el apoyo a proyectos de investigación y creación artística de la Universidad Nacional de Colombia 2017-2018, a la convocatoria 617 Doctorados Nacionales 2014 de Colciencias y al Programa Reconstrucción del Tejido Social en Zonas de Posconflicto en Colombia del Proyecto Modelo ecosistémico de mejoramiento rural y construcción de paz: Instalación de 
capacidades locales. Financiado por el Fondo Nacional de Financiamiento para la Ciencia, la Tecnología y la Innovación, Fondo Francisco José de Caldas contrato n. ${ }^{\circ}$ 213-2018 Código 58960.

\section{REFERENCIAS}

[1] M. Reza Khanzadi, "Phase Noise in Communication Systems Modeling, Compensation, and Peformance Analysis", PhD disseation, Chalmers University of Technology - Göteborg, Sweden, 2015.

[2] A. F. Betancur-Pérez, J. J. Granada Torres y N. Guerrero González, "Redes elásticas del futuro: beneficios para la red interna de telecomunicaciones de Colombia”,Tecno Lógicas, vol. 18 , n. 34 , pp. $125-136,2015$. DOI: $10.22430 / 22565337.222$

[3] N. J. Gomes, M. Morant, A. Alphones, B. Cabon, J. E. Mitchell, C. Lethien, M. Csörnyei, A. Stöhr y S. Iezekiel, "Radio-over-fiber transport for the support of wireless broadband services”, J. Opt. Netw., vol. 8, pp. 156-178, 2009. DOI: 10.1364/JON.8.000156

[4] R. D. Gallego Ruiz, Y. D. Sánchez Sierra, J. J. Granada Torres, A. M. Cárdenas Soto y N. Guerrero González, "Compensación de los efectos dispersivos de la fibra óptica en un sistema IM-DD usando ecualización LMS y MLSE”,Rev. Investig. - Univ. del Quindio, vol. 27, n. ${ }^{\circ}$ 2, pp. 40-47, 2015. DOI: 10.33975/riuq.vol27n2.53

[5] A. García Marqués, "Cuantificación del estado del canal para la minimización de la potencia en sistemas con transmisores adaptativos", $\mathrm{PhD}$ dissertation, Universidad Carlos III de Madrid - Madrid, Spain, 2007.

[6] Umesha G. B.y M. N. Shanmukha Swamy, "Survey on Different Types of Channel Estimation Techniques in MIMO-OFDM Communication Systems",Int. J. Eng. Technol. Manag. Appl. Sci., vol. 5, n. ${ }^{\circ}$ 1, pp. 15-19, 2017. http://www.ijetmas.com/admin/resources/project/paper/ f201701041483534241.pdf

[7] Z. Zhao, X. Cheng, M. Wen, B. Jiao y C.-X. Wang, “Channel Estimation Schemes for IEEE 802.11p Standard”,IEEE Intelligent Transportation System Magazine, vol. 31, n. ${ }^{\circ}$ 9, pp. 3531-3540, 2013. DOI: 10.1109/MITS.2013.2270032

[8] C. Prieto del Amo, "Estimación de canal y desplazamiento de frecuencia en sistemas MIMO-OFDM con prefijo cíclico insuficiente", PhD dissertation, Universidad Carlos III de Madrid - Madrid, Spain, 2015.

[9] H. Kaur, M. Khosla y R. K. Sarin, "Channel Estimation in MIMO-OFDM System: A Review", in Proceedings of the 2nd International Conference on Electronics, Communication and Aerospace Technology, ICECA 2018, pp. 974-980. DOI: 10.1109/ICECA.2018.8474747

[10] P. Sure y C. M. Bhuma, "A survey on OFDM channel estimation techniques based on denoising strategies",Eng. Sci. Technol. an Int. J., vol. 20, n. ${ }^{\circ}$ 2, pp. 629-636, 2017. DOI: 10.1016/j.jestch.2016.09.011 
[11] A. M. Jaradat, J. M. Hamamreh y H. Arslan, "Modulation Options for OFDM-Based Waveforms: Classification, Comparison, and Future Directions", IEEE Access, vol. 7, pp. 17263-17278, 2019. DOI: 10.1109/ACCESS.2019.2895958

[12] I. Fernández de Jauregui Ruiz, "Advanced modulation formats and nonlinear mitigation for spectral efficient optical transmission systems", $\mathrm{PhD}$ dissertation, Institut National des Télécommunications - Paris, France, 2018. DOI: 10.1109/JLT.2008.927778

[13] A. J. Viterbi y A. M. Viterbi, "Nonlinear estimation of PSK-modulated carrier phase with application to burst digital transmission",IEEE Trans. Inf. Theory, vol. IT-29, n. ${ }^{\circ}$, pp. 543-551, 1983. DOI: 10.1109/TIT.1983.1056713

[14] M. G. Taylor, "Phase estimation methods for optical coherent detection using digital signal processing”, J. Light. Technol., vol. 27, n. 7, pp. 901-914, 2009.

[15] Z. Tao, L. Li, L. Liu, W. Yan, H. Nakashima, T. Tanimura, S. Oda, T. Hoshida y J. C. Rasmussen, "Improvements to Digital Carrier Phase Recovery Algorithm for HighPerformance Optical Coherent Receivers",IEEE J. Sel. Top. Quantum Electron., vol. 16, n. ${ }^{\circ}$ 5, pp. 1201-1209, 2010. DOI: 10.1109/JSTQE.2010.2040370

[16] R. Schmogrow, R. Bouziane, M. Meyer, P. A. Milder, P. C. Schindler y R. I. Killey, "Real-time OFDM or Nyquist pulse generation - which performs better with limited resources?",Opt. Express, vol. 20, n. 26 , pp. B543-B551, 2012. DOI: 10.1364/OE.20.00B543

[17] I. Slim, C. Xie and J. A. Nossek, "Orthogonal Frequency Division Multiplexing for Spectrally Efficient Nyquist WDM Systems," OFDM 2014; 18th International OFDM Workshop 2014 (InOWo'14), Essen, Germany, 2014, pp. 1-4. URL: http://ieeexplore.ieee.org/stamp/stamp.js $\mathrm{p} ? \mathrm{tp}=$ \&arnumber $=6924252 \&$ isnumber $=6924248$

[18] X. Liu, C. Sethumadhavan, T. Pfau y G. Gavioli, "Detection and Removal of Cycle Slip from Received Signals", United States Patent Application 20150215050, 2015. [Internet]. Disponible: http://www.freepatentsonline.com/y2015/0215050.html

[19] J. A. Hartigan y M. A. Wong, "Algorithm AS 136 : A K-Means Clustering Algorithm", J. R. Stat. Soc. Ser. C Applied Stat., vol. 28, n. 1, pp. 100-108, 1979.

[20] N. Guerrero González, D. Zibar, A. Caballero y I. Tafur Monroy, "Experimental 2.5-Gb/s QPSK WDM phase-modulated radio-over-fiber link with digital demodulation by a K -means algorithm",IEEE Photonics Technol. Lett., vol. 22, n. ${ }^{\circ}$ 5, pp. 335-337, 2010. DOI: 10.1109/ LPT.2009.2039640 